EUROPEAN ORGANIZATION FOR NUCLEAR RESEARCH

European Laboratory for Particle Physics

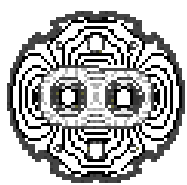

Large Hadron Collider Project

LHC Project Report 830

\title{
Optics Flexibility and Matching at LHC Injection
}

\author{
O. Brüning, H. Burkhardt, B. Goddard, V. Kain, V. Mertens, A. Verdier, T. Risselada \\ CERN, Geneva, Switzerland
}

\begin{abstract}
An excellent match between the SPS, the several kilometres long transfer lines and the LHC will be required to minimise emittance blow-up at injection. Several optics changes in the SPS and the LHC injection insertions had to be accommodated in the design phase. The new 3-phase collimation system in the transfer lines results in additional phase advance constraints. It will be important to maintain some tuning range for the LHC commissioning phase and to accommodate possible further optics changes. We analyse the requirements, the constraints, the current status and options to enhance the optics flexibility.
\end{abstract}

Presented at

PAC 2005, Knoxville, USA, May 16-20, 2005

CERN, CH-1211 Geneva 23,

Switzerland

Geneva, June 2005 


\title{
Optics Flexibility and Matching at LHC Injection
}

\author{
H. Burkhardt, O. Brüning, B. Goddard, V. Kain, V. Mertens, T. Risselada, A. Verdier \\ CERN, Geneva, Switzerland
}

\begin{abstract}
An excellent match between the SPS, the several kilometres long transfer lines and the LHC will be required to minimise emittance blow-up at injection. Several optics changes in the SPS and the LHC injection insertions had to be accommodated in the design phase. The new 3-phase collimation system in the transfer lines results in additional phase advance constraints. It will be important to maintain some tuning range for the LHC commissioning phase and to accommodate possible further optics changes. We analyse the requirements, the constraints, the current status and options to enhance the optics flexibility.
\end{abstract}

\section{INTRODUCTION}

Any emittance blow-up at injection into the LHC will directly reduce the luminosity. The LHC design allows for an only $7 \%$ emittance increase ( 3.5 to $3.75 \mu \mathrm{m}$ in normalized emittance) from extraction in the SPS up to collisions in the LHC. In consequence, an excellent matching at injection is required for the LHC.

Restrictions on particle losses, aperture and beam halo re-population further constrain the already tight requirements on the injection optics. The geometrical emittance in the LHC is largest at injection and the physical aperture very tight $(7.5 \sigma)$.

\section{PRECISION IN INJECTION MATCHING}

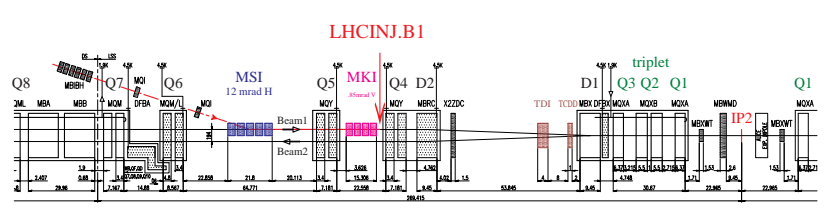

Figure 1: Left side of IR2 with the injection region for LHC ring 1.

Beams are injected from the SPS through two each about $2.5 \mathrm{~km}$ long transfer lines, TI 2 and TI 8 , into the LHC. At the end of the transfer lines to the LHC, the incoming beams are horizontally deflected in the septum magnets (MSI) and finally vertically deflected on the LHC orbit by the injection kickers (MKI). For quantitative estimates, we refer here specifically to a matching point (LHCINJ.B1 in Fig. 1), halfway between the kicker MKI and the closest downstream quadrupole Q4. At this point, the $\beta$ functions are about $60 \mathrm{~m}$ and the dispersions of order $D=10 \mathrm{~cm}$, in both rings and transverse planes $(x, y)$.

To remain within the total $7 \%$ emittance budget from the SPS up to LHC collisions, we aim at individual contributions not exceeding the $2 \%$ level [1]. This implies roughly that $\beta$ functions should be matched between the SPS, the transfer lines and the LHC to a level of roughly $10 \%$. For dispersions $D$ we aim at matching to about $10 \mathrm{~cm}$ and $2 \mathrm{mrad}$ in $D^{\prime}$. For a more detailed discussion of the expected emittance growth at injection based on calculations, measurements and simulation, we refer to another contribution at this conference [2].

The transfer lines start and end with matching sections equipped with individually powered quadrupoles, to allow to adapt to changes in the SPS and LHC optics. We will now describe which SPS and LHC optics changes can be anticipated and then discuss the required and available optics flexibility.

\section{OPTICS CHANGES}

On the SPS-side, the most significant optics change in the last years was that the working point was lowered from roughly 26.6 to 26.2 (similar in $x, y$ ) which resulted in a $3 \%$ change in $\beta_{x, y}$ at extraction and about $10 \mathrm{~cm}$ in dispersion. Both transfer lines start with SPS-matching sections which have the flexibility to adapt to changes of this size.

More flexibility is required on the LHC side. Several optics versions exist or can be anticipated and are discussed below. In addition, there are extra constraints on phase advance differences between the transfer line collimators, which were added at a later stage [3] to the transfer line design [4] for protection purposes.

\section{1) Rematch transfer lines to LHC optics V6.5}

There has been a change in the LHC layout to allow for extra non-linear correctors. The Q3 magnets had to be moved by $0.3 \mathrm{~m}$ towards the IP in several insertions, IR $1,2,5$ and 8 .

Table 1: Comparison of optics parameters at LHCINJ.B2 between optics versions 6.4 and 6.5 (sep. bumps off).

\begin{tabular}{|ccccccc|}
\hline & $\begin{array}{c}\beta_{x} \\
\mathrm{~m}\end{array}$ & $\alpha_{x}$ & $\begin{array}{c}D_{x} \\
\mathrm{~m}\end{array}$ & $\begin{array}{c}\beta_{y} \\
\mathrm{~m}\end{array}$ & $\alpha_{y}$ & $\begin{array}{c}D_{y} \\
\mathrm{~m}\end{array}$ \\
\hline V6.4 & 49.13 & -1.69 & -0.11 & 84.93 & 1.24 & 0.00 \\
V6.5 & 53.23 & -1.73 & -0.11 & 75.20 & 1.22 & 0.00 \\
\hline
\end{tabular}

For injection, this implied a change of about $\pm 10 \%$ in $\beta_{x, y}$ between the previous and actual optics version, see Table 1. The strengths of the Q5 magnets which are common to the LHC and the transfer lines, also changed by about $10 \%$. The rematch of the transfer line optics to the modified LHC optics implied significant changes in the optimum transfer line collimator positions. Together with 
other, cost saving arguments, this resulted in a modification of the transfer line collimation with a repositioning of a few transfer line collimators [5].

The LHC layout is now frozen. Major changes like the Q3 move are not expected in the future.
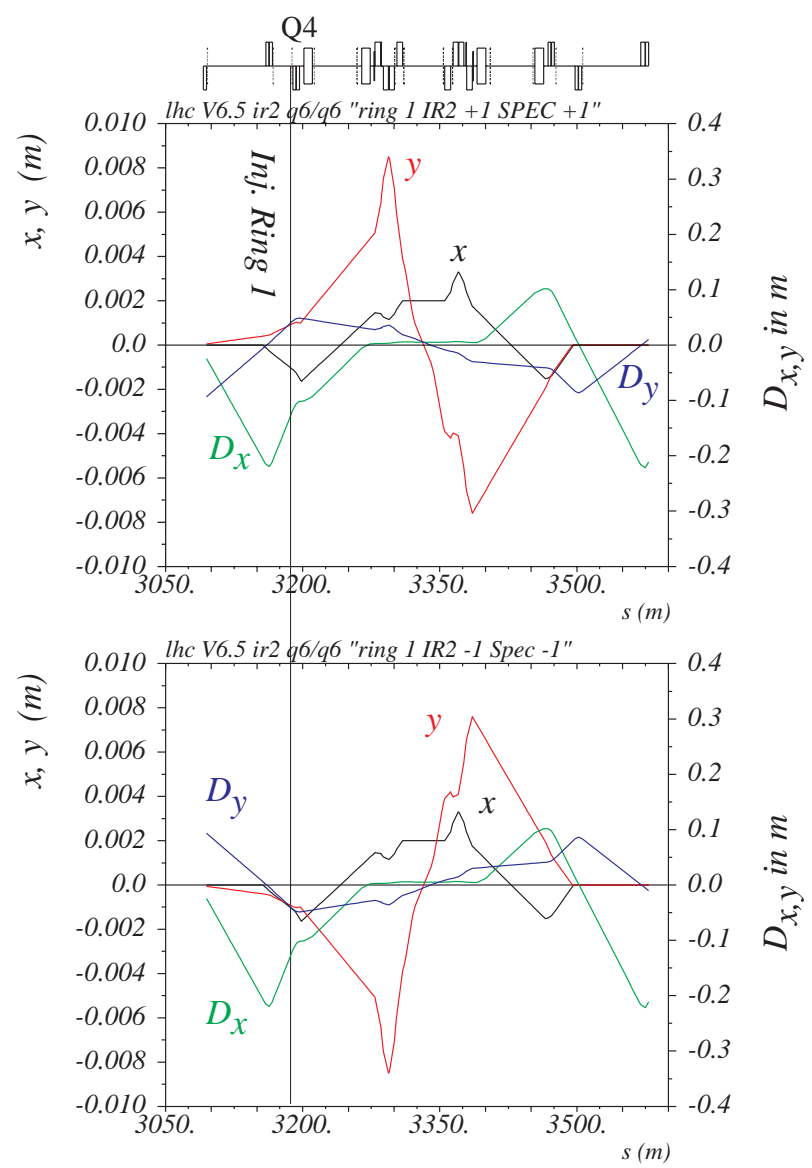

Figure 2: Orbit and dispersion for positive (top) and negative (bottom) vertical crossing angles at IR2.

\section{2) Crossing angle configurations}

The experiments which share the insertions used for injection (ALICE in IR2, LHC-B in IR 8) will require different crossing angles and spectrometer polarities. A summary of the various configurations can be found in the LHC Design report [6]. The spectrometer bumps are closed within the Q1 magnet positions left and right of the interaction point (IP) and should not affect the injection at Q4. The external crossing bumps are closed within Q6-Q6 and do not change $\beta$-functions (to first order). They change the orbit and dispersion, as can be seen in Fig. 2 for IR2.

The expected range of dispersion at injection in IR2 is $D_{x}=-11$ to $-15 \mathrm{~cm}$ and $D_{y}= \pm 5 \mathrm{~cm}$ as a result of polarity changes of the vertical crossing angle within $\pm 170 \mu \mathrm{rad}$.

For injection in ring 2 at IR8, the crossing angle is in the horizontal plane, see Fig.3.

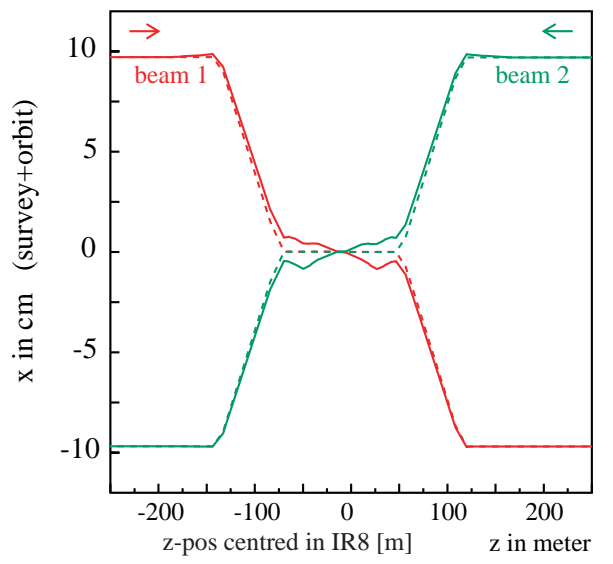

Figure 3: Horizontal beam positions in IR8 with (solid) and without (dashed line) horizontal crossing at IP 8.

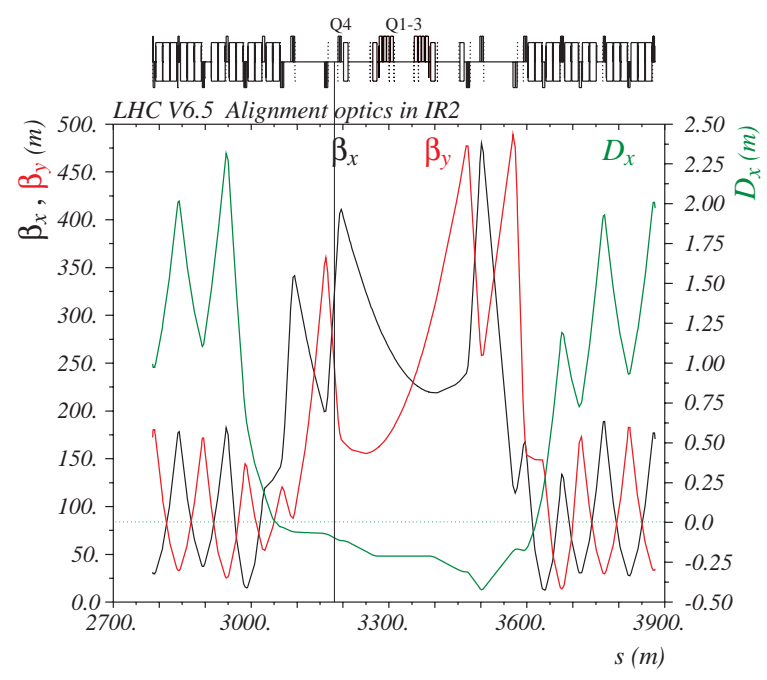

Figure 4: IR2 alignment optics.

Negative external crossing angles would interfere with the geometrical separation between ring 1 and 2 and are not foreseen. The range of dispersion for injection at IR8 to consider is $D_{x}=-6$ to $-11 \mathrm{~cm}$ and $D_{y}=0$ to $0.4 \mathrm{~cm}$ from zero horizontal crossing angle up to $+170 \mu \mathrm{rad}$.

\section{3) LHC working point. Integer tune changes}

The tunes of the design optics for the LHC are $Q_{x, y}=$ $64.28,59.31$, with an integer tune difference of $\Delta Q=$ 5. A possible alternative could be the "resonance-free" lattices [6], with $Q_{x, y}=59.28,51.31$ or $Q_{x, y}=$ $67.28,59.31$, both with $\Delta Q=8$. Integer tune changes in this range can be achieved in the LHC using the arc quadrupoles and it is possible to match the LHC insertions using the quadrupoles Q6 to Q13 in a way which is transparent for injection [7].

\section{4) Alignment optics}

Special alignment optics, in which the triplet quadrupoles Q1-Q3 are turned off have been studied 
for the low $\beta$ insertions IR1, IR5 and more recently also been considered for IR2, see Fig. 4.

The $\beta$ functions at injection close to $\mathrm{Q} 4$ increase by about a factor of five compared to the standard optics and the $90^{\circ}$ phase advance between the kicker and TDI is lost (reduced to $\Delta \mu_{y} \approx 20^{\circ}$ ). This is not suitable for injection, except maybe for very low intensity pencil beams for special machine studies.

It should however be possible to completely avoid injection into an IR with alignment optics. Measurements with the alignment optics in IR2 can be done using beam 2 injected with the standard optics in IR8 and vice versa.

\section{5) Change of $\beta^{*}$ at injection}

We currently foresee $\beta^{*}=10 \mathrm{~m}$ in IR 2,8 at injection. Alternatives, with an increase to $\beta^{*}=18 \mathrm{~m}$ as planned for IR1 5, have been investigated [8]. It was found that the $\beta$ function at the injection points scale approximately linear with $\beta^{*}$. A change from $\beta^{*}=10 \mathrm{~m}$ to $\beta^{*}=18 \mathrm{~m}$ implies an $80 \%$ increase in $\beta$ at injection and a re-match would require hardware modifications.

\section{6) Match to the real LHC optics with magnet and alignment errors}

We assume that large optics and alignment errors will already have been corrected in the LHC, to a level of about $20 \%$ in $\beta$ and $10 \%$ in residual dispersion (about $12 \mathrm{~cm}$ at injection). A first level of corrections in the LHC at injection will primarily be driven by aperture considerations rather then luminosity as magnet errors will change with the ramp and squeeze.

\section{EXISTING AND ENHANCED FLEXIBILITY}

The matching sections at the end of the transfer lines have each ten individually powered quadrupoles. They are sufficient to allow changes on the level of $20 \%$ in $\beta_{x, y}$ and $10 \mathrm{~cm}$ in dispersion, as required to adapt to the real LHC optics and changes in crossing angle. The phase advance between the transfer line collimators, which will be installed in these sections, will however also be affected. Several cases have been studied and up to $15^{\circ}$ change in phase advance for $20 \%$ modification in $\beta$ and about $10^{\circ}$ for changes in dispersion on the order of $10 \mathrm{~cm}$ were observed. This implies a loss in the protected aperture of the order of $10 \%$ (to be verified by tracking).

Several options to enhance the flexibility exist and are being considered.

Dispersion matching can in principle also be achieved using orbit correctors. There has been good experience in LEP using orbit correctors to minimise dispersion, with the possibility of dispersion free steering [9]. A potential advantage in using orbit correctors rather than quadrupoles to match dispersion is that this would to first order de-couple dispersion and $\beta$-function matching. It could be a good solution for the small dispersion changes depending on the various crossing angle schemes.

The optics flexibility can also be enhanced using more individually powered quadrupoles. The loss in protected aperture due to changes in phase advance can be compensated by adding further transfer line collimators. A comparative study of the potential and cost of various options is planned.

\section{SUMMARY}

Excellent optics matching at injection is important for the LHC. A flexibility of about $20 \%$ in $\beta$-functions and $10 \mathrm{~cm}$ in dispersion is required to be able to adapt to crossing angles and to match to the real LHC machine including errors and tolerances. This is feasible with the present transfer lines but implies compromises in collimation. The transfer line collimators are located in the LHC matching sections which couples $\beta$-functions and phase advance between collimators. A $20 \%$ change in $\beta$ can cause a change in phase advance differences of $15^{\circ}$ and reduce the protected aperture by about $10 \%$.

Much larger changes in $\beta$ (for example by a factor of 1.8 to follow the change of $\beta^{*}$ from 10 to $18 \mathrm{~m}$ ) cannot be expected to be matched without hardware changes.

The alignment optics should not be used in the insertion in which the beam is injected.

Several options to enhance the flexibility exist and are being studied. This includes the use of orbit correctors to match dispersion and studies to enhance the flexibility with more individually powered quadrupoles.

\section{REFERENCES}

[1] H. Burkhardt, "Optics Flexibility and Matching at LHC Injection", LHC project workshop proceedings, CERN-AB-2005-014 DI p. $168 \mathrm{ff}$.

[2] B. Goddard, H. Burkhardt, V. Kain, and T. Risselada, "Expected Emittance Growth and Beam Tail Repopulation from Errors at Injection into the LHC", this conference.

[3] H. Burkhardt, "Do we need collimation in the transfer lines ?", Chamonix XII proceedings, p. 190, CERN AB/2003-008 ADM (2003).

[4] A. Hilaire, V. Mertens, and E. Weisse, "Beam Transfer to and Injection into LHC", LHC Project Report 208 and Proc. EPAC 1998, pp.2117-2119.

[5] H. Burkhardt, B. Goddard, Y. Kadi, V. Kain, T. Risselada, and W. Weterings, "Collimation in the Transfer Lines to the LHC”, this conference.

[6] “The LHC design report, Vol.I Chapter 4”, CERN-2004-003.

[7] A. Verdier, priv. comm.

[8] A. Lombardi, priv. comm.

[9] R. Assmann, P. Raimondi, G. Roy, and J. Wenninger, "Emittance optimization with dispersion free steering at LEP”, Phys. Rev. ST Accel. Beams 3 (2000) 121001. 